\title{
Residence time distribution and flow field study of aero-shielded solar cyclone reactor for emission-free generation of hydrogen
}

\author{
Vidyasagar Shilapuram, D. Jaya Krishna ${ }^{1}$, Nesrin Ozalp* \\ Texas A\&M University at Qatar, Mechanical Engineering Department, PO Box 23874, Doha, Qatar
}

\section{A R T I C L E I N F O}

\section{Article history:}

Received 28 May 2011

Received in revised form

5 August 2011

Accepted 11 August 2011

Available online 1 September 2011

Keywords:

Solar reactor

Residence time

Methane decomposition

Hydrogen

CFD
A B S T R A C T
This paper provides a thorough analysis on the flow field and Residence Time Distribution
(RTD) of our "aero-shielded cyclone solar reactor" designed to generate hydrogen from
solar thermal methane cracking process. The analysis has been carried out based on the
results from flow dynamics, and residence time distribution by using Computational Fluid
Dynamics (CFD). Kinetics is taken from the literature and the reactor volume is estimated
based on a plug flow reactor assumption. Residence time distribution characteristics are
obtained by gas tracer injection method, and particle tracking method. Based on the results
of our flow studies, "reactors in series model" is adopted to model the aero-shielded
cyclone reactor. Path lines show that operating variables have significant effect on the
flow behavior inside the reactor. Results show that thermo chemical properties of the gases
have effect on the flow behavior which significantly affect the mean residence time in the
reactor. Results also show that the residence time, spread of the tracer by variance, and the
number of reactors in series are observed to be changed by change in the flow rate, type of
screening gas, and methane mole fraction in the feed.
Copyright $\odot 2011$, Hydrogen Energy Publications, LLC. Published by Elsevier Ltd. All rights reserved.

\section{Introduction}

Solar thermal cracking of methane is a promising hydrogen production technique because of its zero emission footprint $[1,2]$. Literature on the kinetics of solar methane decomposition can be categorized into two groups: (1) when there is no carbon in the feed gas, and (2) when the feed gas is laden with carbon [3]. For example, Rodat et al. [4] studied the kinetics of methane decomposition in a tubular solar reactor using Dsmoke software. They obtained a kinetic expression for the overall dissociation reaction from the reactor model assuming a plug flow and non-catalytical reaction. On the other hand,
Wyss et al. [5] obtained the best fit kinetic parameters by minimizing the sum of squares of the residuals for methane conversions determined experimentally and theoretically. Another example study on the kinetics of methane decomposition with no carbon in the feed gas was done by Sinaki et al. [6]. As for the literature on the kinetics of methane decomposition using carbon particles, most of them state that reaction order is 0.5 and it does not change when different carbon samples are used [7-12]. Conversely, Trommer et al. [13] assumed methane decomposition as a first order and estimated the kinetic parameters accordingly. More details and discussions on the kinetic parameters found in literature

\footnotetext{
* Corresponding author. Tel.: +974 6686 2832; fax: +974 44230066.

E-mail address: nesrin.ozalp@qatar.tamu.edu (N. Ozalp).

${ }^{1}$ Present address: Mechanical Engineering Department, BITS Pilani - Hyderabad Campus, Hyderabad 500078, India. 0360-3199/\$ - see front matter Copyright @ 2011, Hydrogen Energy Publications, LLC. Published by Elsevier Ltd. All rights reserved. doi:10.1016/j.ijhydene.2011.08.035
} 
for methane decomposition when carbon particles are laden with methane, and when there is only methane in the feed gas can be found elsewhere [3].

Several reactor concepts have been tested to achieve improved efficiency and reduced carbon deposition in solar thermal methane cracking reactors. For example, a laboratory scale nozzle-type solar reactor based on direct heating concept was developed for methane decomposition by Abanades and Flamant [14]. Graphite nozzles in various geometries were tested to see the effects on methane decomposition. Results showed that the nozzle geometry is one of the main parameters for efficient methane decomposition because it affects the residence time, radiation adsorption efficiency and gas-solid flow dynamics. They observed that pyrocarbon formation on the reactor wall accumulates over time and finally block the reactor at the entrance of the nozzle. In a follow up study, Abanades and Flamant used argon as the carrier gas, which was injected at the top of the pyrex glass window, to prevent the carbon particle deposition [15]. After these studies, they tested indirect heating solar concept predicting that pyrocarbon formation can be avoided by temperature homogenization [16]. Finally, they developed a medium-scale multi tubular double walled solar reactor $(10 \mathrm{~kW})$ based on the indirect heating reactor concept [4,17-20]. Experiments were conducted to determine the reactor performance as a function of operating conditions. Kinetic simulations were carried out using Dsmoke software. As a further study, $50 \mathrm{~kW}$ multi-tubular solar reactor was constructed, tested and simulated to acquire more experience toward an industrial scale [21]. Abanades and Flamant [17] and Rodat et al. [22] stated that solar reactors based on indirect heating provides advantage over the solar reactors based on direct heating concept. They further stated that the solar irradiation zone is separated from the reacting flow zone and therefore particles do not deposit on the window. A recent study by Rodat et al. suggested maintaining turbulent flow inside the solar reactor for large scale systems to minimize the transport limitations (heat and mass transfer) [23]. As for the carbon deposition issue inside the solar reactor for large scale systems, two possible solutions were proposed: either to use reactor material that can withstand oxidation (alumina is used in industry), or apply mechanical cleaning.

Another good reactor concept, so called "fluid-wall aerosol reactor", was developed by a group of researchers at Colorado University [24]. Their experiments were successfully carried out for the methane decomposition in their fluid-wall reactor consisting of concentric tubes made of porous graphite instead of solid tube wall used by other research groups $[5,25]$. The reactant methane is sent in the innermost porous graphite tube where methane cracking takes place resulting with carbon formation. The purpose of porous tube was to create an aerodynamic blanket for preventing carbon black deposition on the inner wall of the reactor tube. Their experimental results suggested using secondary concentrator to further augment the solar flux from primary concentrators to achieve high temperatures inside the reactor. Intrinsic kinetic and sensitivity analysis were carried out using experimental data $[26,27]$. In a later study, they re-designed their reactor to reduce the reradiation losses and to increase the thermal efficiency with a new concept, which was consists of three concentric vertical tubes with sunlight reflected toward the reactor through a secondary concentrator [28]. They conducted experiments to study the effect of temperature, and initial methane flow rate on the conversion with and without carbon black co-feed. The carbon black products were analyzed using TEM. However their experiments showed that deposition of the carbon black particles is observed in the hot zone of the reactor when the reactor is operated for long time.

Kogan group of Weizmann Institute of Science developed two reactor concepts with "tornado flow". Tests were performed in unseeded reactor at atmospheric pressure and at temperatures up to $1320 \mathrm{~K}$ [29]. They achieved $28 \%$ methane conversion. As a further study, room temperature seeding simulation tests were performed for different reactor bottom configuration at various injection locations below window surface for different primary, secondary and tertiary flows. The objective was to prevent reactor window contamination by contact with the solid particles. The streamlined design of the reactor solved the problem of particle deposition on reactor window [30]. They made a qualitative comparison of various cases using CFD (Computational Fluid Dynamics) simulation to predict experiment results [31]. Although they eliminated carbon deposition on the window and reduced on reactor walls, their reactor concept still had the carbon clogging due to accumulation at the exit.

A $5 \mathrm{~kW}$ "vortex flow reactor" was designed, fabricated and tested by Steinfeld research group at ETH-Zurich [32,33]. Tests were performed for different combinations, such as; mounting the reactor vertical and horizontally, inlet port at the front side and the rear side, with and without graphite inner cavity etc. They achieved a maximum methane conversion of $98.8 \%$ and hydrogen yield of $99.1 \%$.

In summary, all of the novel reactor concepts by different research groups have demonstrated significant effort and improvement toward seeking solution for reactor clogging, and carbon contamination, as well as they focused on enhancement of the heat transfer, reduction of kinetic limitations, obtain uniform temperature, and effectively utilize the solar radiation by various reactor configurations with direct or indirect heat transfer concepts. They all came to a conclusion that production of hydrogen rich gas with continuous removal of all carbon formed, without deposition of carbon in any location of the reactor, is the biggest challenge in methane cracking solar reactor technology. Therefore, carbon deposition and reactor clogging problem still remains as one of the major motivation to conduct research in solar thermal methane cracking field. In our paper, we present the results of our efforts on searching a solution to this problem with our new reactor concept, named "aero-shielded solar cyclone reactor".

\section{Current state of the computational studies on methane cracking solar reactors}

CFD has become a powerful tool to conduct reaction study simulations to design a reactor in addition to observe flow field, mixing behavior, temperature profiles, and concentration distribution. There are several major computational studies done by the above research groups to characterize flow field, temperature distribution etc. inside their methane 
cracking solar reactors. For example, Abanades et al. presented gas and reactor temperature profiles, axial gas velocity, chemical conversion, and molar concentration of methane in their nozzle-type laboratory scale solar reactor using Femlab 3.1 software [15]. Mass and energy transport with chemical reaction kinetics were coupled in the computational fluid dynamic model. First order reaction kinetics with kinetic constants adopted from Trommer et al. [13]. The simulation result showed that high temperature gradients exist between nozzle center and reactor wall. Very narrow region on the reactor wall was utilized for methane decomposition. In order to increase the reaction surface, a graphite slab was inserted. Results showed that the reacting gas is heated more homogenously when the temperature gradients is smaller. In another study, Abanades et al. also performed experimental and theoretical study for cavity type tubular reactor [17]. This time, their computational flow model was based on finite volume incorporating fluid flow, heat and mass transfer, and chemical reaction using Fluent 6.2. The two phase flow with 2D axisymmetrical CFD modeling determined the hydrogen concentration and velocity at reactor outlet, velocity and temperature profiles inside the reactor, and methane conversion. Later on, the same group carried out a 3-D CFD simulation of nonsymmetrical reactor geometry to predict the temperature distribution in their cavity type tubular reactor and to simulate thermophoretic carbon deposition on reactor wall [18]. In one of their recent studies, they did thermal simulations of $53 \mathrm{~kW}$ solar reactor using Fluent 12.0.16 [21]. They observed that majority of the incoming power were lost due to high temperature gradient through the water cooled aluminum front face. In order to solve this problem, optimized design of front face was proposed and simulated which improved the absorption efficiency.

Weimer group of Colorado University developed 2-D axisymmetric model by considering only heat transfer and fluid flow to study the temperature and velocity profiles of the flow in a their solar thermal fluid-wall aerosol flow reactor, e.g. kinetics is not included $[5,25]$. Their objective was to create an aerodynamic blanket on inner porous graphite reactor tube wall to prevent carbon black clogging. However, when the aerosol flow solar reactor was operated for a long time period deposition of carbon black was observed on the porous tube wall in the reactor hot zone. Simulations were done using two different gases namely argon and hydrogen as the annular sweeping gas. Higher temperatures were obtained toward the center of core region when hydrogen is used as the sweeping gas.

Kogan et al. conducted the preliminary CFD simulations of the tornado flow solar reactor without including the kinetics [31]. Comparison of their experimental results against the predictions from their CFD work was qualitative in nature. However, CFD simulation helped them to clarify some intricate features of the flow under study.

Recently, Ozalp and Kanjirakat presented a numerical validation to the experimental results of Kogan group, and provided a thorough parametric study by using computational fluid dynamic analysis on the effect of inlet angles, main flow rate, screening flow rate, screening gases, and particle deposition [34]. The study concluded that the application of discrete phase model with particle tracking successfully predicts particle deposition in a solar thermal methane cracking reactor. Furthermore, as a continuation of that study, effect of carbon particle seeding for the improvement of solar reactor performance was studied [35]. They simulated the gasparticle flow inside the tornado flow reactor via Lagrangian discrete phase model. It was observed that the carbon particle seeding inside the reactor considerably increased the temperature inside the reactor.

Another study by Ozalp and Devanuri [36] investigated a numerical validation to the experimental results of vortex flow reactor of Hirsch and Steinfeld [32]. Results were presented in terms of outlet temperatures, contours for static temperature and concentration of chemical species. Simulations proved that the radiative heat transfer mechanism is the dominant means of heat transfer compared to the effects of conduction and convection.

\subsection{Residence Time Distribution (RTD) analysis by CFD}

Residence Time Distribution (RTD) analysis is a very important concept to characterize mixing and flow behavior inside a reactor, and to know whether the reactor is approaching any of an ideal reactor: plug flow reactor or mixed flow reactor. Also, RTD analysis helps to model the real reactor as a combination of ideal reactors. In addition, RTD data can also be used to analyze any non-idealities like channeling, by passing, and short circuiting present in a reactor. By fitting the RTD data to appropriate models, the results give the model parameters. This eventually can be used to scale up or to design a reactor once the reaction kinetics is obtained [37].

Swirling flows are common to increase residence time and stabilize the flow pattern in combustion. Rotating flows are also encountered in variety of applications like in turbo machinery, mixing tanks, etc [38]. These swirls cause to observe distribution in residence time of each fluid element. In addition, Holmen et al. [39] mentioned that residence time will have the effect on product gas composition. It may be observed from the above literature that this approach has been well applied in other research areas [40-44], but till date there is no RTD analysis by CFD in solar methane cracking field except for our previous work in Ref. [45]. Therefore, in the present study, "reactors in series model" is chosen to model our aero-shielded solar cyclone reactor.

\section{Methodology}

\subsection{Chemical kinetics}

Traditionally, chemical kinetics of methane decomposition is determined experimentally by conducting the reaction at constant temperature in a laboratory scale reactor. In this study, we have adopted the kinetics of Trommer et al. [13] and then determined the reactor volume as $2.6 \mathrm{~L}$ by following this methodology:

(i) Assume methane feed rate going into the process,

(ii) Assume the extent of conversion of the reactant/yield of the product gas you desire at the assumed reaction temperature, 
(iii) Estimate the residence time by assuming plug flow performance based on the kinetics obtained via aforementioned methodology.

(iv) From the estimated residence time in Step (iii) and methane feed rate/yield of hydrogen chosen in Step (i), the reactor volume is estimated.

\subsection{CFD analysis}

In order to understand the flow behavior we have applied our validated CFD model [34-36] to our aero-shielded reactor concept shown in Fig. 1. The three-dimensional geometry for simulations is built and a non-uniform unstructured grid is generated using GAMBIT. The details of the problem geometry are provided in Fig. 1 . The basic geometry with adapted grid used for simulations is shown in Fig. 2.

The reactor is provided with main flow of methane (F1) and window screening flow (F2) as shown in Fig. 1. According to the reactor conditions, the flow is considered to be isothermal with species transfer. In this concept, methane is injected through 18 impeller disk jets with each of $2 \mathrm{~mm}$ diameter from the top center of the reactor with a $45^{\circ}$ angle at different flow rates as provided in Table 1 . The objective is to create vortex flow of main gas (methane) in the center without interfering to the walls in order to prevent carbon black deposition. As for the vortex flow inside the reactor, the purpose is to increase the residence time of methane to achieve higher methane conversion.

It may be noted that the reaction is not considered in the simulations since the present study is to observe the flow field and the mixing behavior. Since methane decomposition is significant at elevated temperatures of above $700{ }^{\circ} \mathrm{C}$, thermal

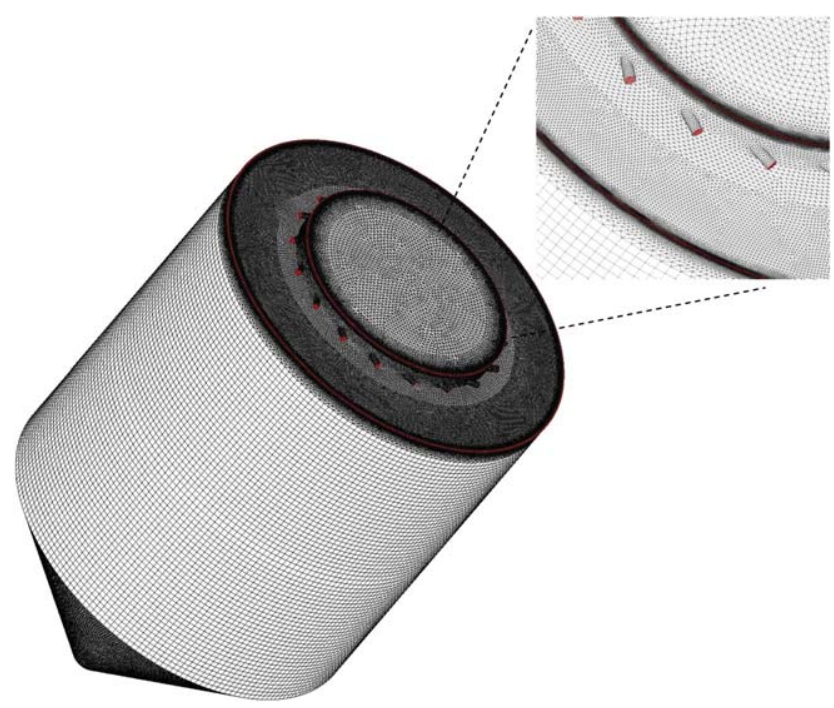

Fig. 2 - Meshed geometry of aero-shielded solar cyclone reactor.

and transport properties were taken at $1000^{\circ} \mathrm{C}$ for simulations of the present study. A commercial finite volume based tool, Ansys 13.0 is employed for our simulations. These simulations involve fluid flow, turbulent species transport and particle tracking. To evaluate the turbulent quantities, RNG $k-\varepsilon$ turbulence model has been employed. The criteria for the selection of RNG $k-\varepsilon$ has been discussed and may be referred to $[34,36]$. As the present study is based on low Reynolds number and swirling flows the RNG $k-\varepsilon$ turbulence model has been a

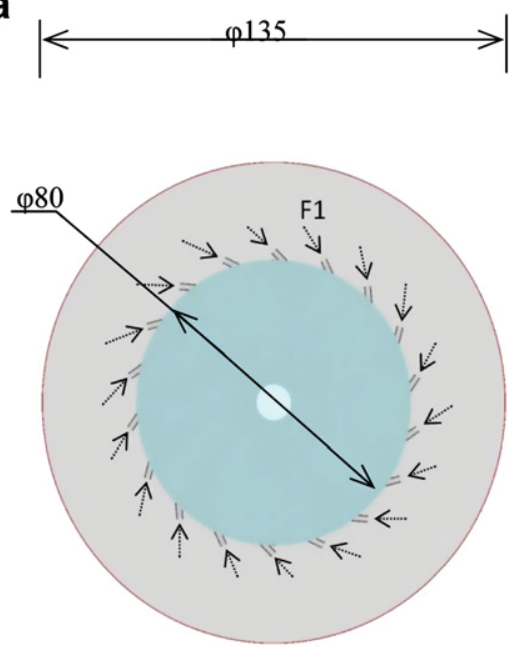

b

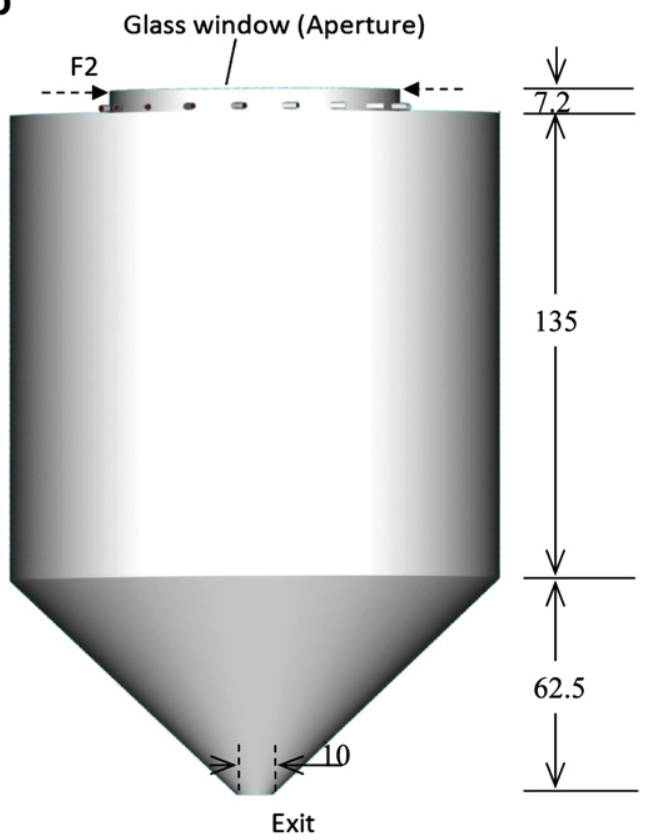

All dimensions in $\mathrm{mm}$

Fig. 1 - Aero-shielded solar cyclone reactor (a) top view (b) front view. 
Table 1 - RTD characteristics by various RTD methods used in FLUENT.

\begin{tabular}{|c|c|c|c|c|c|c|c|c|c|}
\hline \multirow[t]{2}{*}{ S.No } & \multirow[t]{2}{*}{$(\mathrm{v})_{0} \mathrm{l} / \mathrm{min}$} & \multirow[t]{2}{*}{$(y)_{\mathrm{CH}_{4}}$} & \multicolumn{3}{|c|}{ Gas tracer method } & \multicolumn{2}{|c|}{ Particle tracking method } & \multirow[t]{2}{*}{ Space time } & \multirow[t]{2}{*}{ Screening gas } \\
\hline & & & $\bar{t}$ & $\sigma^{2}$ & $\mathrm{~N}$ & $\bar{t}$ & $\sigma^{2}$ & & \\
\hline 1 & 1 & 1 & 149.4 & 7258 & 3 & 185.8 & 217.1 & 135.7 & - \\
\hline 2 & 10 & 1 & 14.3 & 82.7 & 2.5 & 19.7 & 21.8 & 13.5 & - \\
\hline 3 & 1 & 0.1 & 157.8 & 15228.1 & 1.6 & 70 & 11.9 & 135.7 & $\mathrm{Ar}$ \\
\hline 4 & 1 & 0.9 & 189.1 & 27,003 & 1.3 & 167 & 162 & 135.7 & Ar \\
\hline 5 & 11.11 & 0.1 & 22.2 & 636.8 & 0.8 & 9.3 & 1.91 & 12.2 & $\mathrm{Ar}$ \\
\hline 6 & 11.11 & 0.9 & 12.5 & 109.6 & 1.4 & 13.9 & 12.2 & 12.2 & $\mathrm{Ar}$ \\
\hline 7 & 1 & 0.1 & 389.3 & 101326.2 & 1.5 & 92 & 21.8 & 135.7 & $\mathrm{H}_{2}$ \\
\hline 8 & 1 & 0.9 & 158.1 & 20,215 & 1.2 & 161.2 & 153.7 & 135.7 & $\mathrm{H}_{2}$ \\
\hline 9 & 11.11 & 0.1 & 12 & 49.86 & 2.9 & 6.5 & 0.535 & 12.2 & $\mathrm{H}_{2}$ \\
\hline 10 & 11.11 & 0.9 & 13.1 & 68 & 2.5 & 14.2 & 81,081 & 12.2 & $\mathrm{H}_{2}$ \\
\hline
\end{tabular}

selected. The equations pertaining to present study are as follows.

Continuity equation:

$\frac{\partial \rho}{\partial t}+\frac{\partial\left(\rho u_{i}\right)}{\partial x_{i}}=0$

Momentum Equation:

$\frac{\partial\left(\rho u_{i}\right)}{\partial t}+\frac{\partial\left(\rho u_{i} u_{j}\right)}{\partial x_{i}}=\frac{\partial P_{\text {eff }}}{\partial x_{i}}+\frac{\partial}{\partial x_{j}}\left[\mu_{\text {eff }}\left(\frac{\partial u_{i}}{\partial x_{j}}+\frac{\partial u_{j}}{\partial x_{j}}\right)\right]-\rho g_{i}$

k-Equation:

$\frac{\partial(\rho k)}{\partial t}+\frac{\partial\left(\rho k u_{\mathrm{i}}\right)}{\partial x_{\mathrm{i}}}=\frac{\partial}{\partial x_{\mathrm{j}}}\left(\left(\mu+\frac{\mu_{\mathrm{t}}}{\sigma_{\mathrm{k}}}\right) \frac{\partial k}{\partial x_{\mathrm{j}}}\right)+G_{\mathrm{k}}-\rho \varepsilon$

$\varepsilon$-Equation:

$\frac{\partial(\rho \varepsilon)}{\partial t}+\frac{\partial\left(\rho \varepsilon u_{i}\right)}{\partial x_{i}}=\frac{\partial}{\partial x_{j}}\left(\left(\mu+\frac{\mu_{\mathrm{t}}}{\sigma_{\varepsilon}}\right) \frac{\partial \varepsilon}{\partial x_{j}}\right)+C_{1 \varepsilon} \frac{\varepsilon}{k} G_{k}-C_{2 \varepsilon} \rho \frac{\varepsilon^{2}}{k}-R_{\varepsilon}$

Species transport equation:

$\frac{\partial\left(\rho Y_{i}\right)}{\partial t}+\frac{\partial\left(\rho u_{j} Y_{i}\right)}{\partial x_{j}}=\frac{\partial}{\partial x_{j}}\left[\left(\rho D_{i m}+\frac{\mu_{t}}{S c_{t}}\right) \frac{\partial Y_{i}}{\partial x_{j}}\right] i=1,2 \ldots, N-1$

where

$\mu_{\mathrm{eff}}=\mu+\mu_{\mathrm{t}}$

$\mu_{\mathrm{t}}=\rho \mathrm{C}_{\mu} \frac{k^{2}}{\varepsilon}$

$C_{\mu}=0.0845$

$C_{1 \varepsilon}=1.42$

$C_{2 \varepsilon}=1.68$

$C_{3 \varepsilon}=\tan h\left|\frac{u_{3}}{\sqrt{u_{1}^{2}+u_{2}^{2}}}\right|$

$G_{k}=\mu_{\mathrm{t}} S_{\mathrm{ij}} \frac{\partial u_{\mathrm{i}}}{\partial x_{\mathrm{j}}}$

$\mathrm{R}_{\varepsilon}=\frac{\mathrm{C}_{\mu} \rho \eta^{3}\left(1-\eta / \eta_{\mathrm{o}}\right)}{1+\beta \eta^{3}} \frac{\varepsilon^{2}}{k}$
$\eta=\frac{S k}{\varepsilon}, S=\left(2 S_{i j} S_{i j}\right)^{1 / 2}, \eta_{\mathrm{o}}=4.38, \beta=0.012$

$S_{i j}=\frac{\left(\frac{\partial u_{i}}{\partial x_{j}}+\frac{\partial u_{j}}{\partial x_{i}}\right)}{2}$

$\sigma_{\mathrm{k}}=0.7179, \sigma_{\varepsilon}=0.7179$

$\rho=\frac{1}{\sum \frac{Y_{\mathrm{i}}}{\rho_{\mathrm{i}}}}$

$\mu=\sum \mathrm{Y}_{\mathrm{i}} \mu_{\mathrm{i}}$

Gas tracer transport equation:

To perform RTD simulations the transient analysis of a tracer has been considered with the same physical properties as that of continuous phase. The transport equation for the concentration of a tracer in a turbulent flow can be given as

$\frac{\partial\left(\rho_{\mathrm{m}} Y_{\mathrm{tr}}\right)}{\partial t}+\frac{\partial\left(\rho_{\mathrm{m}} u_{\mathrm{m}} Y_{\mathrm{tr}}\right)}{\partial x_{j}}=\frac{\partial}{\partial x_{j}}\left[\left(\rho_{\mathrm{m}} D_{\mathrm{im}}+\frac{\mu_{\mathrm{t}}}{S c_{\mathrm{t}}}\right) \frac{\partial Y_{\mathrm{tr}}}{\partial x_{\mathrm{j}}}\right]$

Particle tracking transport equation:

Another approach by which RTD simulations can be carried out is by particle tracking method. A DPM (Discrete phase model) is used to evaluate the particle trajectory through the continuous phase of gas. The motion of the particulate phase is done by integrating the force balance on the particle in Lagrangian reference frame [38]. The particle trajectory is thus calculated as follows

$\frac{\mathrm{d} u_{\mathrm{p}, \mathrm{i}}}{\mathrm{d} t}=F_{\mathrm{D}}\left(u_{\mathrm{i}}-u_{\mathrm{p}, \mathrm{i}}\right)+\frac{g_{\mathrm{i}}\left(\rho_{\mathrm{p}}-\rho\right)}{\rho_{\mathrm{p}}}$

where $F_{\mathrm{D}}=\left(18 \mu / \rho_{\mathrm{p}} d_{\mathrm{p}}^{2}\right) C_{\mathrm{D}} \operatorname{Re}_{\mathrm{p}, \mathrm{i}} / 24$ and the drag coefficient $C_{\mathrm{D}}$ is as given in [38]. The Reynolds number may be given as Eq. (9).

$\operatorname{Re}_{\mathrm{p}, \mathrm{i}}=\frac{\rho_{\mathrm{p}} d_{\mathrm{p}}\left|u_{\mathrm{p}, \mathrm{i}}-\mathrm{u}\right|}{\mu}$

The governing equations are solved by using finite volume method. SIMPLE algorithm has been employed for pressure velocity coupling. The convective terms of the momentum, species, turbulent kinetic energy and turbulent dissipation 
rate equations are discretized using second order upwinding scheme. To ensure the results obtained by the numerical study are independent of the computational grid, grid independence studies are carried out. A grid size of 3,346,656 cells has been considered for the study as further increase in computational grid is observed to have insignificant effect on the solution.

\subsection{Steps followed to perform CFD simulations for RTD}

\subsubsection{Gas tracer injection method}

1. Initially, run the steady state simulations for the flow rates as provided in Table 1. It may be noted that the first term in left hand side of Eqs. (1-5) $\left(\partial \rho / \partial t, \partial\left(\rho u_{i}\right) / \partial t, \partial(\rho k) / \partial t, \partial(\rho \varepsilon) / \partial t\right.$ and $\left.\partial\left(\rho Y_{i}\right) / \partial t\right)$, boils down to zero due to steady state condition.

2. Once the convergence is achieved for the steady state simulations, the species mass fraction for the tracer is made one and the mass fraction for other species is made zero.

3. As the tracer needs to follow the path of the main flow the transport properties of the tracer are given as that of methane.

4. Now the transient equation for the tracer (Eq. (7)) with a time step of 0.1 is carried out for one iteration. It is to be noted that at this time step the Eqs. (1-5) should be deactivated.

5. After step 4, the species mass fraction for the tracer is made zero and mass fraction for other species should be changed to that of conditions as provided in step 1.

6. Now the flow equations (Eqs. (1-7)) are solved by plotting the tracer concentration at the exit with respect to time.

7. Collect the concentration verses time data at the exit of the reactor.

It is assumed that a tracer does not undergo any chemical reaction with the reactants or products. The tracer amount is very small and all the physical properties of the tracer are practically same as the working fluid. This way, the flow is not disturbed inside the reactor after the tracer is introduced. Assumptions made by Nauman [46] in his recent study are used in our RTD studies. RTD characteristics such as mean residence time, variance, and exit age distribution are calculated. A reactor in series model is chosen to find out the number of ideal reactors from inverse of dimensionless variance.

\subsubsection{Particle tracking method}

1. Initially, run the steady state simulations for the flow rates provided in Table 1 . It may be noted that the first term in left hand side of Eqs. (1-5) $\left(\partial \rho / \partial t, \partial\left(\rho u_{i}\right) / \partial t, \partial(\rho k) / \partial t, \partial(\rho \varepsilon) / \partial t\right.$ and $\left.\partial\left(\rho Y_{i}\right) / \partial t\right)$ boils down to zero due to steady state condition.

2. Once the convergence is achieved for the steady state simulations, inject the particle as a tracer with a diameter of $10^{-6} \mathrm{~m}$ and which has similar fluid properties as that of methane. This procedure ensures that the particles can perfectly follow all the simulated time scales.

3. Discrete phase model is used to evaluate the particle trajectory through the continuous phase of gas.

4. Then the residence time is recorded at the reactor outlet as each particle tracer exits from the outlet.

5. Obtain the histogram of time taken by the particles leaving the reactor, mean residence time, number of particles injected, number of particles tracked.

\subsection{Validation of the adopted methodology}

Hirsch and Steinfeld [32] carried out experiments for methane decomposition in their vortex flow solar reactor. They observed that the mean residence time for runs \#7 and \#8 is $7.3 \mathrm{~s}$ (as presented in Table 3 of their study). They estimated the residence time from experimentally obtained conversion by assuming plug flow with first order methane decomposition. However, residence time is independent of reaction kinetics, but depends on reaction stiochiometry for elementary reactions. Usually, mean residence time based on reactor entrance condition and exit condition is calculated as $\mathrm{V}_{\text {reactor }} / \nu_{\mathrm{O}}$ and $\mathrm{V}_{\text {reactor }} / \nu_{\mathrm{O}}\left(1+\alpha \mathrm{X}_{\mathrm{CH}_{4}}\right)$ respectively. Reactor exit condition includes the volume expansion as a result of reaction as well as inert gas mole fraction. An alternative method of experimental measurement in terms of operating variable can be found in our previous study [45]. As shown in Table 2, for runs \#7 and \#8, it is estimated that mean residence time is $4.95 \mathrm{~s}$ based on entrance conditions, while mean residence time is 4.63 and $4.7 \mathrm{~s}$ based on reactor exit conditions. But experimentally noted mean residence time $(7.3 \mathrm{~s})$ is considerably much higher than the residence time estimated based on entrance and exit conditions. This confirms that vortex flow exists in their reactor geometry and as a result of this more residence time is noticed.

In order to validate the present RTD methodology the residence time distribution by CFD simulations are performed for vortex flow solar thermal reactor of Hirsch and Steinfeld [32]. Gas tracer method and particle tracking method has been employed to study the residence time distribution and mean residence time. The results thus obtained are presented in Table 2. It may be observed that the results from the present study for mean residence time obtained by two different CFD

Table 2 - Comparison of experimentally observed mean residence time for the run \#7 and \#8 of Table 3 in Hirsch and Steinfeld [32] with present study.

\begin{tabular}{|c|c|c|c|c|c|c|c|c|}
\hline S.No. & $\begin{array}{c}\text { Run } \\
\text { (Hirsch and } \\
\text { Steinfeld [32]) }\end{array}$ & $\begin{array}{c}\text { Total flow } \\
\text { rate, } \\
l_{\mathrm{n}} / \mathrm{min}\end{array}$ & $\begin{array}{l}\text { Methane } \\
\text { mole } \\
\text { fraction }\end{array}$ & $\begin{array}{l}\text { Estimated based } \\
\text { on reactor } \\
\text { entrance } \\
\text { condition, s }\end{array}$ & $\begin{array}{l}\text { Estimated } \\
\text { based on } \\
\text { reactor exit } \\
\text { condition, s }\end{array}$ & $\begin{array}{c}\text { Experimentally } \\
\text { observed, s } \\
\text { (Hirsch and } \\
\text { Steinfeld [32]) }\end{array}$ & $\begin{array}{l}\text { Gas tracer } \\
\text { method } \\
\text { (Present } \\
\text { study) }\end{array}$ & $\begin{array}{c}\text { Particle } \\
\text { tracking } \\
\text { method } \\
\text { (Present study) }\end{array}$ \\
\hline 1 & $\# 7$ & 19 & 0.158 & 4.95 & 4.63 & 7.3 & 7.78 & 8.81 \\
\hline 2 & $\# 8$ & 19 & 0.789 & 4.95 & 4.70 & 7.3 & 8.18 & 9.02 \\
\hline
\end{tabular}


based RTD methods are in good agreement with the experimentally observed mean residence time. This confirms the validation of our RTD by CFD methodology for the vortex flow reactor used for methane decomposition. Same methodology has been adopted for our reactor called "Aero-shielded solar cyclone reactor" which was designed and developed in house for methane decomposition to produce hydrogen.

\section{Results and discussions}

\subsection{Flow field}

Previous studies report that only argon, helium and nitrogen have been used as screening gas. In our study, we tested hydrogen as the screening gas in addition to the argon and made a comparison. Figs. 3-5 show the flow field with the path lines marked by particle ID for the following cases: (i) when there is no window screening, (ii) argon as a screening gas, and (iii) hydrogen as a screening gas for the same total flow rate. From these results, it can be observed that there is a significant difference in the flow field when different screening gases are used.

\subsection{RTD by gas tracer method}

Once the steady flow field is attained inside the reactor as shown in Figs. 3-5, a gas tracer is injected as a pulse into the reactor. Simultaneously, concentration of the tracer is measured at the exit of the reactor as a function of time. RTD by gas tracer and RTD by particle tracking are performed covering a wide range of flow rates as shown in Table 1.In this
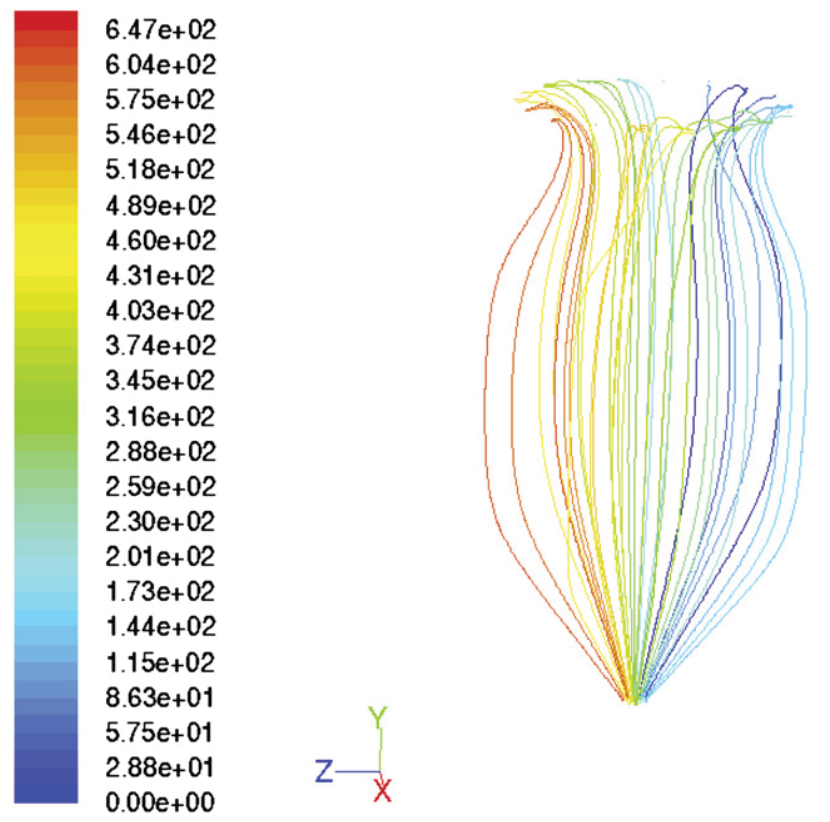

Pathlines Colored by Particle ID

Fig. 3 - Flow field in aero-shielded reactor with no screening gas.

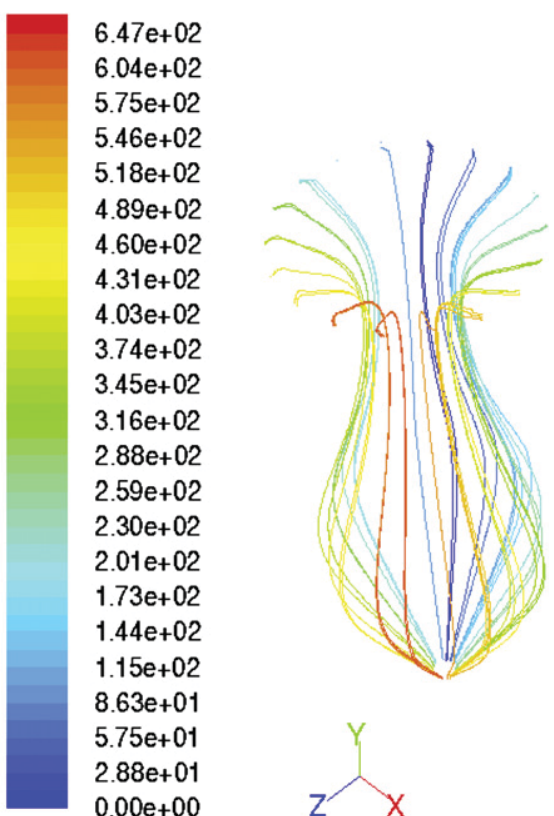

Pathlines Colored by Particle ID

Fig. 4 - Flow field in aero-shielded reactor with argon as screening gas.

study methane is considered as feeding gas and, hydrogen or argon are considered as screening gases. The concentration at the exit is measured and the residence time characteristics are calculated as shown below.

Mean residence time:

$\bar{t}=\frac{\sum C_{i} t_{i} \Delta t_{i}}{\sum C_{i} \Delta t_{i}}$

Variance:

$\sigma_{\mathrm{t}}^{2}=\frac{\sum \mathrm{t}_{\mathrm{i}}^{2} \mathrm{C}_{\mathrm{i}} \Delta \mathrm{t}_{\mathrm{i}}}{\sum \mathrm{C}_{\mathrm{i}} \Delta \mathrm{t}_{\mathrm{i}}}-\overline{\mathrm{t}}^{2}$

Exit age distribution:

$E(t)=\frac{C(t)}{\sum C_{i} \Delta t_{i}}$

Dimensionless residence time:

$\theta=\frac{t}{\bar{t}}$

Dimensionless variance is given by

$\sigma_{\theta}^{2}=\frac{\sigma_{\mathrm{t}}^{2}}{\overline{\mathrm{t}}^{2}}$

Dimensionless exit age distribution:

$E_{\theta}=\bar{t} E$ 


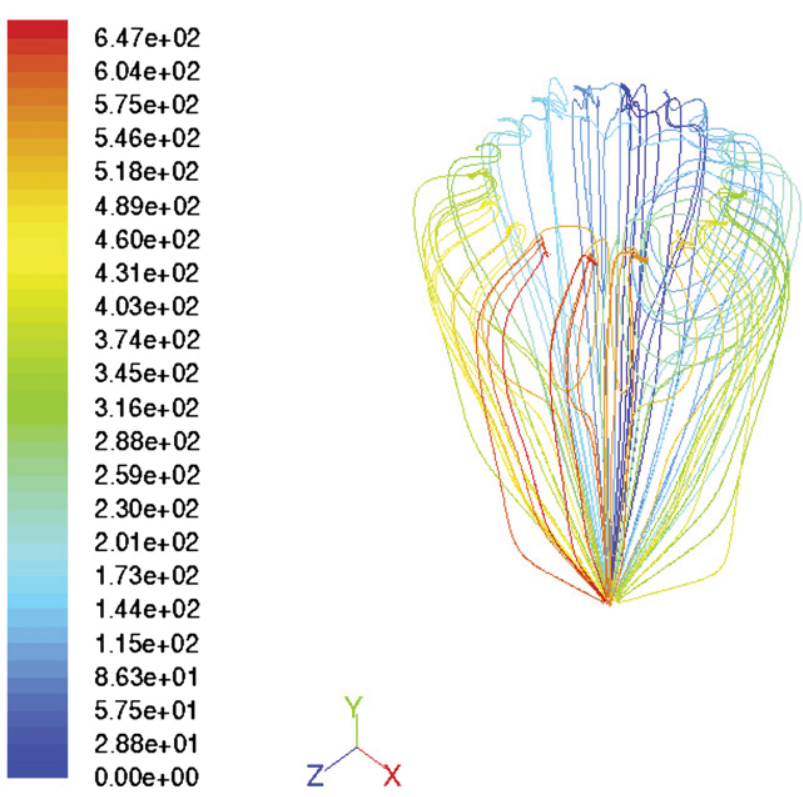

\section{Pathlines Colored by Particle ID}

Fig. 5 - Flow field in aero-shielded reactor with hydrogen as screening gas.

Reactors in series model is adopted to obtain the number of reactors. For the pulse input, tracer mass balance for $\mathrm{N}$ reactors in series resulted in the following equation

$E_{\theta}=(N \bar{t}) E=N \frac{(N \theta)^{N-1}}{(N-1) !} e^{-N \theta}$

For each run the exit age distribution is calculated from concentration verses time data. Then number of reactors in series is obtained by fitting Eq. (16) with the exit age distribution data. The other means to calculate the number of reactors in series is from the inverse of dimensionless variance, which is followed in the present study.

\subsection{Flow analysis in vortex or cyclone reactors}

Let's consider two cases with same reactor volume, e.g. same length and diameter. The first reactor is a plug flow reactor as shown in Fig. 6(a), whereas the other reactor is a vortex or swirl flow reactor as shown in Fig. 6(b). In plug flow reactor of Fig. 6(a), each fluid element has the same residence time $t$ and has a flat velocity profile. On the other hand, vortex flow reactor reactants are injected tangentially, which creates swirls as shown in Fig. 6(b). Fluid elements travel with different length of swirls with different length of time, i.e., $t_{1}$, $t_{2}, t_{3}$, and $t_{4}$. If we make a comparison, for the same reactor volume, the fluid element in (b) takes more time than the fluid element in (a) i.e., $\left(t_{1}+t_{2}+t_{3}+t_{4}>t\right)$. Therefore, it would give inaccurate performance parameters if the flow in (b) is taken as a plug flow. Essentially, since the residence time of the fluid element in (b) is bigger, higher extent of reaction and thereby more hydrogen yield is expected compared to residence time $t$ of (a). Therefore, performance parameters of solar reactors with swirling flows should be modeled as follows. (i) In (c), plug flow reactor model with residence time $\tau$, which is equal to $t_{1}+t_{2}+t_{3}+t_{4}$, gives the exact estimate compared to residence time $t$.

(ii) In (d), plug flow reactors in series with residence times in each reactor is $t_{1}, t_{2}, t_{3}$ and $t_{4}$, respectively.

(iii) If none of the above applies, then the experimental residence time should be estimated as discussed in [45].

If all the fluid elements across the cross section follow the same path as shown in (b), one can estimate the experimental residence time as mentioned in step (iii). Then the use of plug flow reactor model to predict the reactor performance is appropriate. In most cases, each fluid element travels with different length of swirl with different length of time, but qualitatively similar to (b). Under these conditions, mixed flow assumption may also deviate from the actual scenario. For this situation, particular residence time of each fluid element would result in residence time distribution across the reactor. Therefore, ideal reactors in series model give an insight to model the reactor and for that reason, we have adopted "ideal reactors in series model" to approximate our aero-shielded solar reactor.

Rodat et al. [4] carried out "three reactors in series" approach for their tubular solar reactor. If we refer to their reactor geometry, we can see that there are annular reactors divided into three zones based on temperature. So, each zone is assumed as a "one plug flow reactor." But the problem is: if we closely observe the reactor tubes, half the length of the reactor tube is in graphite cavity, which is in high temperature zone $(>700 \mathrm{~K})$. Remaining length of the reactor tubes are in lower temperatures than $700 \mathrm{~K}$, where inner and outer tubes are each considered as a plug flow reactor. But in reality, methane decomposition will not occur at that low temperature $(<700 \mathrm{~K})$. So, single plug flow reactor assumption with volume (or corresponding space time) equal to the volume inside the inner tube and the volume in the annular region in high temperature zone would be enough. Hence, three plug flow reactors in series approach may not be suitable for their reactor geometry. On the other hand, in our aero-shielded solar reactor, the reactor is in one zone with respect to reaction temperature and the swirls are observed throughout the length of the reactor because of tangential entry. So, the time spent by each swirl should be equivalent to space time of one reactor volume if we want to use the reactors in series approach. However, the flow profile inside the reactor shows that it is neither plug flow nor mixed. Therefore, in our present study, "reactors in series model" is chosen to model our aeroshielded solar reactor. Then a tracer is injected as a pulse input and finally tracer mass balance for the $\mathrm{N}$ reactors in series were done. Essentially, the number of reactors can be obtained either by fitting the experimental concentration verses time data, or from the residence time characteristics of exit age distribution i.e. dimensionless variance [37].

\subsection{Effect of gas flow rate}

Fig. 7 shows the effect of gas flow rate on the residence time distribution of fluid in the reactor. It may be observed that residence time distribution is different for different flow rates. The increase in flow rate results in decrease of mean 
a

b
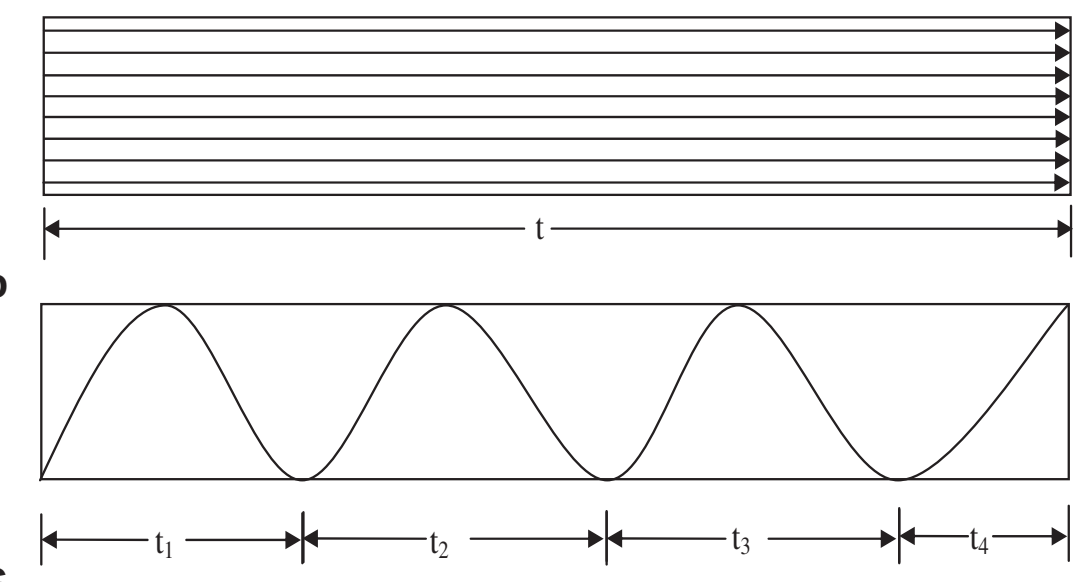

C

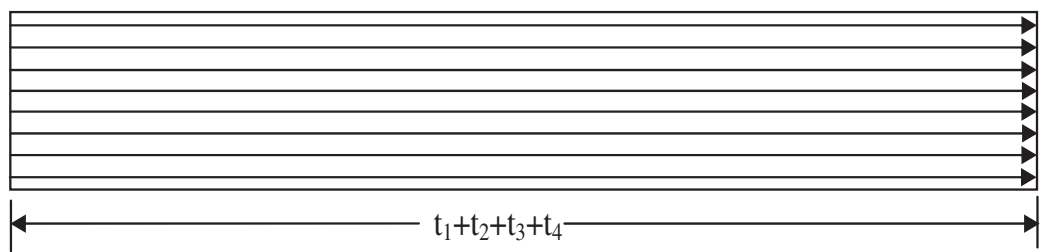

d

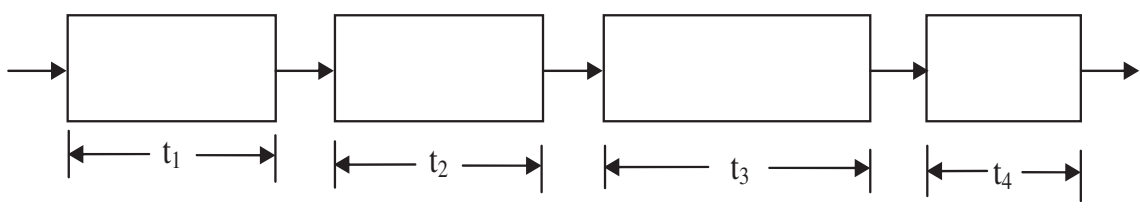

Fig. 6 - Vortex or swirl flow analysis.

residence time in the reactor. The peak of the normalized curve concentration increases with an increase in the flow rate.

\subsection{Effect of the screening gas}

Fig. 8 shows the effect of screening gas when the total flow rate and mole fraction of inert screening gas is constant. From this figure, it can be observed that residence time distribution and mean residence time are different for different screening gas. This is due to the formation of different flow field for different screening gases as explained in Section 4.1 and can be observed from Figs. 3-5. Furthermore, this can also be explained in terms of space time and mean residence time. For a reactor, space time is calculated either based on reactor entrance condition $\left(=V_{\text {reactor }} / \nu_{0}\right)$ or based on reactor exit conditions $\left(=\mathrm{V}_{\text {reactor }} / \nu_{\mathrm{o}}\left(1+\alpha \mathrm{X}_{\mathrm{CH}_{4}}\right)\right)$ where $\alpha$ is the volume expansion factor or inert gas mole fraction in the feed gas, i.e., screening gas in our present situation. So, space time calculated based on reactor exit condition must be same for different screening gases as long as same mole fraction of screening gas is maintained in the feed gas. This is because ' $\alpha$ ' is the mole fraction of inert gas immaterial what gas it is. But the mean residence time calculated from the RTD data showed different mean residence time as shown in Fig. 8 and
Table 1. Therefore, the difference in mean residence time might be due to difference in thermo chemical properties of various screening gases. Similar kind of behavior also observed in our previous study for vortex flow reactor [45]. The spread or distribution of the tracer would be larger when there is no screening gas. The long tail observed with argon as screening gas indicates that there might be a dead volume at some locations inside the reactor, which is also observed with

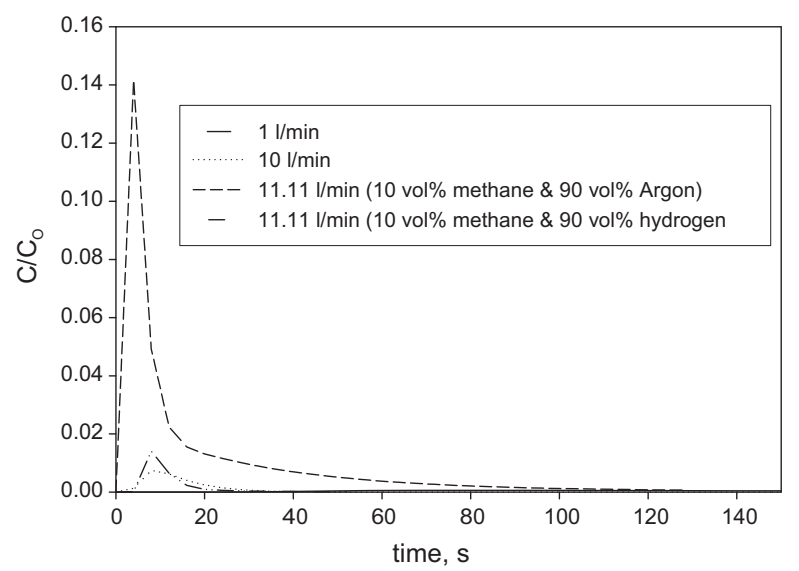

Fig. 7 - Effect of gas flow rate on RTD. 


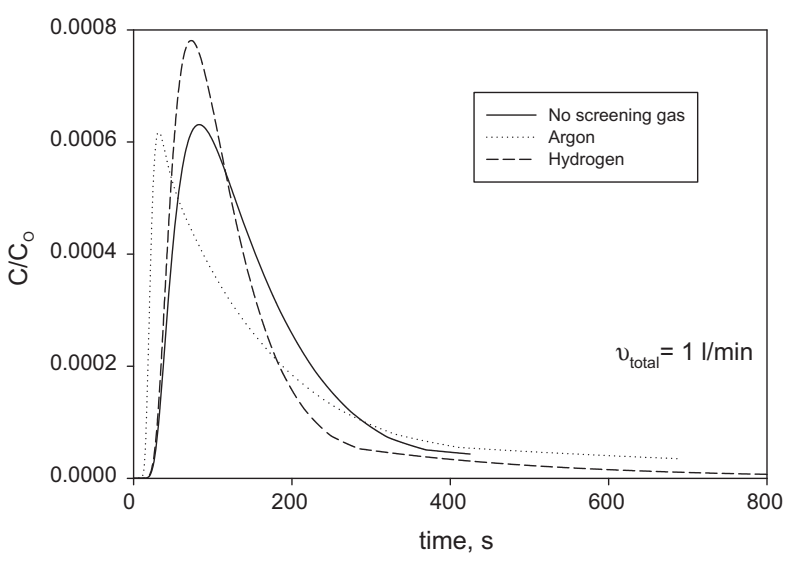

Fig. 8 - Effect of screening gas on RTD.

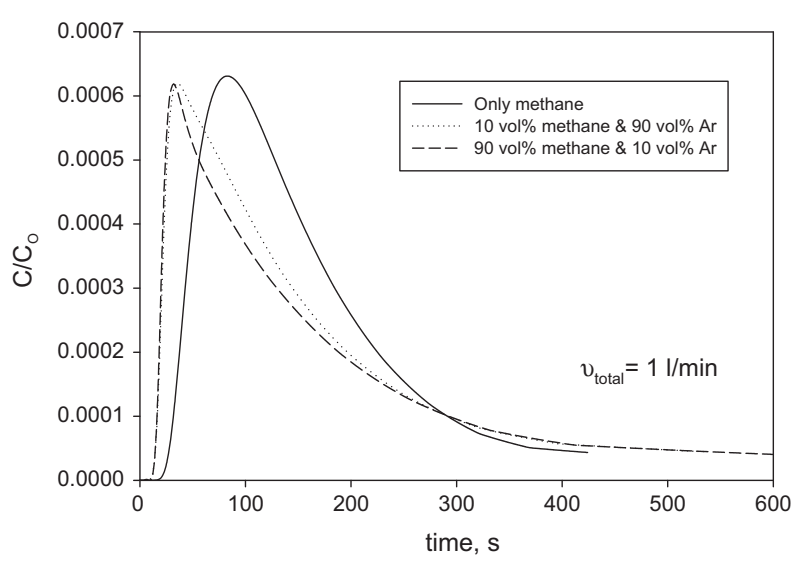

Fig. 9 - Effect of methane mole fraction on RTD. increase in total flow rate. Finally, a very nice peak in tracer concentration is observed when hydrogen is used as the screening gas.

\subsection{Effect of methane mole fraction}

Fig. 9 shows the effect of methane mole fraction in the feed gas with argon as screening gas for total flow rate of $1 \mathrm{l} / \mathrm{min}$. Peak height in concentration ratio is observed to be same independent of methane feed mole fraction, but the residence time distribution and mean residence time observed is different. This shows that, in addition to thermo chemical properties of screening gases as discussed in Section 4.5, the methane mole fraction in the feed gas has significant effect on the flow dynamics and mean residence time.

\subsection{RTD by particle tracking method}

In this method, once the flow becomes steady, which is confirmed by solution convergence in CFD, known number of particles is injected as a pulse at the reactor inlet. Then estimation is made on how much time it takes for each particle to reach the outlet. Fig. 10 shows the histogram of the residence time distribution calculated from the CFD model with particle tracking. This is for a total volumetric feed rate of $11.11 \mathrm{l} / \mathrm{min}$ with $90 \%$ methane by volume and hydrogen as the screening gas. Numbers of particles injected and tracked are 648 and 645, respectively, with mean residence time and standard deviation of $14.2 \mathrm{~s}$ and 81,081 respectively. A comparison of mean residence time by above two methods gives the same residence time within normal limits.

\subsection{Summary of the results}

Table 1 shows a summary of all simulations. Space time in the last before column of Table 1 is calculated based on entrance conditions of the reactor $\left(=\mathrm{V}_{\text {reactor }} / \nu_{\mathrm{o}}\right)$. We can see that there is no regular trend in mean residence time with increase in flow rate, or with type of screening gas, or with increase in mole fraction of methane. However, if we compare mean residence time by various tracer methods and space time, we can see that the mean residence time obtained by various methods change within $\pm 20 \%$ except for few experiments. Except for one case, mean residence time values obtained by gas tracer method are higher than that of space time calculations based on entrance condition of the reactor. Similarly, number reactors in series estimates does not show a regular trend with increase in flow rate, or by different screening gas, or by change in methane mole fraction in feed gas.

Maag et al. [32] experimentally observed that for the same volumetric feed rate, performance parameters were different when (i) methane mole fraction is different, and (ii) difference in specific heat of methane compared to argon. Additionally, Abanades and Flamant [15] noticed bulk gas mixture inside the reactor is heated more efficiently because of difference in thermal conductivity of argon and hydrogen gas i.e., high thermal conductivity of hydrogen compared to argon. They stated that physical properties like density, viscosity, thermal conductivity, and heat capacity of the gas mixture have significant effect on the gas composition and temperature. Furthermore, Dahl et al. [24] concluded that physical properties of gas like viscosity and thermal conductivity has a great

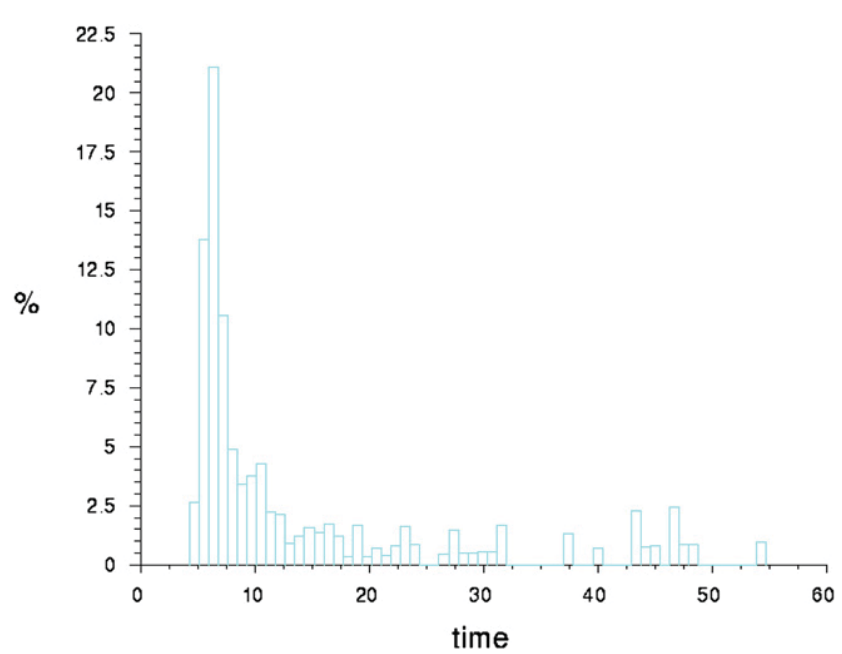

Fig. 10 - RTD histogram by particle tracking method. 
effect on temperature and velocity profiles. Therefore, these studies $[15,24,32]$ reported the effect of thermo chemical properties of gases on temperature and velocity profile, whereas we came to a conclusion based on our RTD analysis that thermo chemical properties of gases have significant effect on the mean residence time as well. The following explanation given below gives an idea on how thermo chemical properties of the screening gas have significant effect on residence time, which is a crucial parameter for the reactor design.

In the present study thermal properties of gases are taken at $1000{ }^{\circ} \mathrm{C}$. The results showed that the mean residence obtained by residence time distribution studies are different under the constant total volumetric feed rate as shown in Table 1 . The reason for this significant difference in flow dynamics is because of different density and viscosity of gases. As a result of this velocity profile and concentration profile will change. This in turn results in change in residence time distribution as well as mean residence time in the vortex or swirl reactor.

\section{Conclusions}

We have characterized the flow behavior of our aero-shielded solar reactor through residence time distribution analysis. The resulting mean residence time and variance via CFD simulations are obtained by two different tracer methods and they are compared with space time based on entrance conditions. Based on the results and discussions, we can draw the following conclusions:

(i) There is significant difference in mean residence time with increase in gas flow rate, type of screening gas and methane mole fraction in the feeding gas.

(ii) The particular trend in estimation of number of ideal reactors are not observed with the operating parameters like gas flow rate, type of screening gas and methane mole fraction in the feeding gas.

(iii) Simulation results proved that the type of screening gas is one of the key parameters in a solar reactor because it determines the flow dynamics, and number of ideal reactor in series.

(iv) Although previous studies reported that the thermo chemical properties of gases have an effect on temperature and velocity profile, our RTD analysis showed that thermo chemical properties of gases have significant effect on the mean residence time as well.

(v) Reactor in series model is more suitable to describe the flow behavior in aero-shielded solar reactor and other methane cracking solar reactors with swirling flow.

(vi) Cyclone or vortex flow inside the aero-shielded solar reactor increases the mean residence time.

\section{Acknowledgment}

This research has been funded by National Priorities Research Program of Qatar National Research Fund (QNRF) Project No:
NPRP 09-671-2-255. Dr. D. Jaya Krishna would like to thank Dr. Nesrin Ozalp for providing summer term research opportunity at TAMUQ.

\section{Nomenclature}

C concentration of tracer, $\mathrm{mol} / \mathrm{m}^{3}$

$C_{1 \varepsilon}, C_{2 \varepsilon}, C_{\mu}, \sigma_{\varepsilon}, \sigma_{\mathrm{k}}$ RNG $k-\varepsilon$ model parameters

d diameter, $\mathrm{m}$

$D_{\text {im }} \quad$ molecular diffusivity, $\mathrm{m}^{2} / \mathrm{s}$

E exit age distribution, $1 / \mathrm{s}$

$E_{\theta} \quad$ exit age distribution in dimensionless form

g gravity, $9.81 \mathrm{~m} / \mathrm{s}^{2}$

$k$ turbulent kinetic energy per unit mass, $\mathrm{m}^{2} / \mathrm{s}^{2}$

$l_{n} \quad$ liters at normal conditions of temperature and pressure

$\dot{n} \quad$ molar flow rate, $\mathrm{mol} / \mathrm{s}$

$\mathrm{N}$ number of ideal reactors

$\mathrm{P} \quad$ pressure, $\mathrm{Pa}$

$\bar{R} \quad$ universal gas constant, $8.314 \mathrm{~J} / \mathrm{mol} \mathrm{K}$

$\mathrm{Re}_{\mathrm{p}} \quad$ relative particle Reynolds number

$\mathrm{Sc}_{\mathrm{t}} \quad$ turbulent Schmidt number

$\mathrm{S}_{\mathrm{ij}} \quad$ mean strain rate tensor

$t \quad$ time, $s$

$\bar{t} \quad$ mean residence time, $s$

$\mathrm{T}$ temperature, $\mathrm{K}$

u velocity, $\mathrm{m} / \mathrm{s}$

$V_{\text {reactor }}$ volume of the reactor, liter

$v_{\mathrm{CH}_{4.0}} \quad$ methane volumetric feed rate, $1 / \mathrm{min}$

$v_{0} \quad$ total volumetric feed rate

$v_{\text {total }}$ total volumetric feed rate

$\mathrm{X}_{\mathrm{CH}_{4}} \quad$ methane conversion

$\mathrm{Y}_{\mathrm{H}_{2}} \quad$ hydrogen yield

$\mathrm{y}_{\mathrm{CH}_{4}} \quad$ mole fraction of methane

$Y_{i} \quad$ local mass fraction

$Y_{\text {tr }} \quad$ tracer mass fraction

\section{Greek letters}

$\rho \quad$ density, $\mathrm{kg} / \mathrm{m}^{3}$

$\rho_{\mathrm{m}} \quad$ density of methane, $\mathrm{kg} / \mathrm{m}^{3}$

$\mu \quad$ viscosity, $\mathrm{kg} / \mathrm{m} / \mathrm{s}$

$\varepsilon \quad$ dissipation rate of turbulent kinetic energy per unit mass, $\mathrm{m}^{2} / \mathrm{s}^{2}$

$\sigma_{\varepsilon}, \sigma_{\mathrm{k}} \quad$ constants in RNG $k-\varepsilon$ model

$\Delta t_{\mathrm{i}} \quad$ difference in successive time steps, $\mathrm{s}$

$\eta \quad$ ratio of turbulence to mean shear time scale

$\eta_{\mathrm{o}} \quad$ RKG $k-\varepsilon$ model parameter

$\sigma^{2} \quad$ variance

$\alpha \quad$ volume expansion due to reaction or inert gas mole fraction in feed

dimensionless residence time

residence time of methane, $\mathrm{s}$

\section{Subscripts}

$\mathrm{i}, \mathrm{j}, \mathrm{k} \quad$ spatial coordinates

p particle

tr tracer

t turbulent

eff effective 


\section{Abbreviations}

RTD Residence Time Distribution
CFD Computational Fluid Dynamics

[18] Rodat S, Abanades S, Sans JL, Flamant G. Hydrogen production from solar thermal dissociation of natural gas: development of a $10 \mathrm{~kW}$ solar chemical reactor prototype. Solar Energy 2009;83:1599-610.

[19] Abanades S, Tescari S, Rodat S, Flamant G. Natural gas pyrolysis in double-walled reactor tubes using thermal plasma or concentrated solar radiation as external heating source. J Nat Gas Chem 2009;18:1-8.

[1] Muradov N, Veziroğlu TN. "Green" path from fossil-based to hydrogen economy: an overview of carbon-neutral technologies. Int J Hydrogen Energy 2008;33:6804-39.

[2] Ozalp N, Kogan A, Epstein M. Solar decomposition of fossil fuels as an option for sustainability. Int J Hydrogen Energy 2009;34:710-20.

[3] Ozalp N, Shilapuram V. Step-by-step methodology of developing a solar reactor for emission-free generation of hydrogen. Int J Hydrogen Energy 2010;35:4484-95.

[4] Rodat S, Abanades S, Coulie J, Flamant G. Kinetic modeling of methane decomposition in a tubular solar reactor. Chem Eng J 2009;146:120-7.

[5] Wyss J, Martinek J, Kerins M, Dahl JK, Weimer A, Lewandowski A, et al. Rapid solar-thermal decarbonization of methane in a fluid-wall aerosol flow reactor fundamentals and application. Int J Chem Reactor Eng 2007; $5: 1-28$.

[6] Sinaki M,Y, Matida EA, Hamdullahpur F. Kinetic model of homogeneous thermal decomposition of methane and ethane. Int J Hydrogen Energy 2009;34:3710-6.

[7] Kim HW, Lee EK, Jun JH, Kong SJ, Han GY, Lee BK, et al. Hydrogen production by catalytic decomposition of methane over activated carbons: kinetic study. Int J Hydrogen Energy 2004;29:187-93.

[8] Pinilla JL, Suelves I, Lazaro MJ, Molieiner R. Kinetic study of the thermal decomposition of methane using carboneous catalysts. Chem Eng J 2008;138:301-6.

[9] Serrano DP, Botas JA, Guil-Lopez R. $\mathrm{H}_{2}$ production from methane pyrolysis over commercial carbon catalysts: kinetic and deactivation study. Int J Hydrogen Energy 2009;34: 4488-94.

[10] Muradov N, Chen Z, Smitha F. Fossil hydrogen with reduced $\mathrm{CO}_{2}$ emission: modeling thermocatalytic decomposition of methane in a fluidized bed of carbon particles. Int J Hydrogen Energy 2005;30:1149-58.

[11] Muradov N. Thermocatalytic $\mathrm{CO}_{2}$-free production of hydrogen from hydrocarbon fuels. Tech report. United State Department Energy; 2003.

[12] Muradov N, Smith F, T-Raissi A. Catalytic activity of carbons for methane decomposition reaction. Catal Today 2005;102: 225-33.

[13] Trommer D, Hirsch D, Steinfeld A. Kinetic investigation of the thermal decomposition of $\mathrm{CH}_{4}$ by direct irradiation of a vortex-flow laden with carbon particles. Int J Hydrogen Energy 2004;29:627-33.

[14] Abanades S, Flamant G. Production of hydrogen by thermal methane splitting in a nozzle-type laboratory-scale solar reactor. Int J Hydrogen Energy 2005;30:843-53.

[15] Abanades S, Flamant G. Solar hydrogen production from the thermal splitting of methane in a high temperature solar chemical reactor. Solar Energy 2006;80:1321-32.

[16] Abanades S, Flamant G. Experimental study and modeling of a high-temperature solar chemical reactor for hydrogen production from methane cracking. Int J Hydrogen Energy 2007;32:1508-15.

[17] Abanades S, Flamant G. High-temperature solar chemical reactors for hydrogen production from natural gas cracking. Chem Eng Commun 2008;195:1159-75.
[20] Rodat S, Abanades S, Flamant G. High-temperature solar methane dissociation in a multitubular cavity-type reactor in the temperature range 1823-2073 K. Energy \& Fuels 2009;23: 2666-74.

[21] Rodat S, Abanades S, Sans JL, Flamant G. A pilot-scale solar reactor for the production of hydrogen and carbon black from methane splitting. Int J Hydrogen Energy 2010;35: 7748-58.

[22] Rodat S, Abanades S, Flamant G. Co-production of hydrogen and carbon black from solar thermal methane splitting in a tubular reactor prototype. Solar Energy 2011;85(4):645-52.

[23] Rodat S, Abanades S, Flamant G. Experimental evaluation of indirect heating tubular reactors for solar methane pyrolysis. Int J Chem Reactor Eng 2010;8:1-19.

[24] Dahl JK, Tamburini J, Weimer A,W. Solar-Thermal processing of methane to produce hydrogen and syngas. Energy \& Fuels 2001;15:1227-32.

[25] Dahl J,K, Weimer A,W, Z'Graggen A, Steinfeld A. Twodimensional axi-symmetric model of a solar-thermal fluidwall aerosol flow reactor. J Solar Energy Eng 2005;127:76-85.

[26] Dahl JK, Barocas VH, Clough DE, Weimer AW. Intrinsic kinetics for rapid decomposition of methane in an aerosol flow reactor. Int J Hydrogen Energy 2002;27:377-86.

[27] Dahl JK, Weimer AW, Krantz WB. Sensitivity analysis of the rapid decomposition of methane in an aerosol flow reactor. Int J Hydrogen Energy 2004;29:57-65.

[28] Dahl JK, Buechler KJ, Weimer AW, Lewandowski A, Bingham C. Solar-thermal dissociation of methane in a fluid-wall aerosol flow reactor. Int J Hydrogen Energy 2004;29:725-36.

[29] Kogan M, Kogan A. Production of hydrogen and carbon by solar thermal methane splitting. I. The unseeded reactor. Int J Hydrogen Energy 2003;28:1187-98.

[30] Kogan A, Kogan M, Barak S. Production of hydrogen and carbon by solar thermal methane splitting. II. Room Hydrogen Energy 2004;29:1227-36.

[31] Kogan A, Israeli M, Alcobi E. Production of hydrogen and carbon by solar thermal methane splitting. IV. Preliminary simulation of a confined tornado flow configuration by computational fluid dynamics. Int J Hydrogen Energy 2007; 32:4800-10.

[32] Hirsch D, Steinfeld A. Solar hydrogen production by thermal decomposition of natural gas using a vortex-flow reactor. Int J Hydrogen Energy 2004;29:47-55.

[33] Maag G, Zanganeh G, Steinfeld A. Solar thermal cracking of methane in a particle-flow reactor for the co-production of hydrogen and carbon. Int J Hydrogen Energy 2009;34: 7676-85.

[34] Ozalp N, Kanjirakat A. Lagrangian characterization of multiphase turbulent flow in a solar reactor for particle deposition prediction. Int J Hydrogen Energy 2010;35:4496-507.

[35] Ozalp N, Kanjirakat A. A CFD study on the effect of carbon particle seeding for the improvement of solar reactor performance. ASME J Heat Transfer 2010;132:122901.

[36] Ozalp N, Devanuri J. CFD analysis on the influence of helical carving in a vortex flow solar reactor. Int J Hydrogen Energy 2010;35:6248-60.

[37] Levenspiel O. Chemical reaction engineering. 3rd ed. New York: John Wiley \& Sons; 1999.

[38] Swirling and rotating flows, fluent6.3 users guide; September 2006. temperature simulation tests of seeded solar reactor. Int J 
[39] Holmen A, Olsvik O, Rokstad OA. Pyrolysis of natural gas: chemistry and process concepts. Fuel Process Technol 1995; 42:249-67.

[40] Moullec Y,L, Potier O, Gentric C, Leclerc J,P. Flow field and residence time distribution simulation of a cross-flow gasliquid wastewater treatment reactor using CFD. Chem Eng Sci 2008;63:2436-49.

[41] Zhang L, Pan Q, Rempel G. Residence time distribution in a multistage agitated contactor with Newtonian fluids: CFD prediction and experimental validation. Ind Eng Chem Res; 2007:3538-46.

[42] Deshmukh SS, Sathe MJ, Joshi JB. Residence time distribution and flow patterns in the single-phase annular region of annular centrifugal extractor. Ind Eng Chem Res 2009;48:37-46.
[43] Bai H, Stephenson A, Jimenez J, Jewell D, Gillis P. Modeling flow and residence time distribution in an industrial-scale reactor with a plunging jet inlet and optional agitation. Chem Eng Res Des 2008;86:1462-76.

[44] Abu-Reesh I,M, Abu-Sharkh B,F. Comparison of axial dispersion and tanks-in-series models for simulating the performance of enzyme reactors. Ind Eng Chem Res 2003;42: 5495-505

[45] Ozalp N, Shilapuram V, Jayakrishna D. Modeling of a vortexflow solar via ideal reactors in series approach. ASME 4 th International conference on energy sustainability. Paper No: ES2010-90324; 2010.

[46] Nauman EB. Residence time theory. Ind Eng Chem Res 2008; 47:3752-66. 